\title{
The predictive value of semaphorins 3 expression in biopsies for biochemical recurrence of patients with low- and intermediate-risk prostate cancer
}

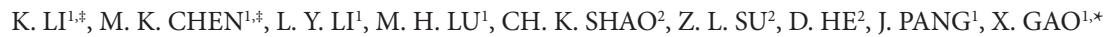 \\ ${ }^{1}$ Department of Urology, The Third Affiliated Hospital, Sun Yat-sen University, Guangzhou, 510630, China; ${ }^{2}$ Department of Pathology, The Third \\ Affiliated Hospital, Sun Yat-sen University, Guangzhou, 510630, China \\ ${ }^{*}$ Correspondence: billurology@126.com \\ ${ }^{*}$ Contributed equally to this work.
}

Received November 12, 2012 / Accepted April 8, 2013

\begin{abstract}
The class-3 semaphorins (Sema3A-F, Sema3s) are initially identified to play an important role in axonal guidance and cell migration. Our previous studies showed that Sema3s are also involved in the lymph node metastasis of prostate cancer, and are likely to modulate the behavior of prostate cancer with a pro-tumoral or an anti-tumoral effect, depending on their subtypes. However, no study has critically investigated the value of Sema3s expression in preoperative biopsy samples for the prediction of biochemical recurrence (BCR) after radical prostatectomy. In this study, we evaluated Sema3s expression by immunohistochemistry on 198 prostate biopsies with low- and intermediate-risk localized prostate cancer. The median follow-up was 42 months (range, 6-60) for all patients. Our results showed that Sema3A (OR: 0.19, $P<0.001$ ), Sema3B (OR: $0.38, P=0.003$ ), Sema3E (OR: $0.39, P=0.007)$, and Sema3C (OR: $2.31, P=0.014)$ staining were independent predictors of $B C R$ on multivariable analysis. Sema3A, $3 \mathrm{~B}, 3 \mathrm{C}$ and $3 \mathrm{E}$ expression demonstrated potential values in predicting $\mathrm{BCR}$ upon survival analysis $(P=0.001, P=0.003, P=0.029, P=0.037$, respectively, Log-rank test). Our findings suggested that Sema3A, $3 \mathrm{~B}, 3 \mathrm{C}$, and $3 \mathrm{E}$ immunostaining in prostate biopsies, as supplements to clinicopathological parameters, could be used for predicting $\mathrm{BCR}$ in low- and intermediate-risk prostate cancer patients after radical prostatectomy. Specially, concurrent Sema3C-positive and Sema3A-negative, 3B-negative, 3E-negative staining is associated with an adverse prognosis. Further prospective studies in larger patient populations are needed to validate the current observations.
\end{abstract}

Key words: biochemical recurrence, biopsy, class-3 semaphorins, immunohistochemistry, prostate cancer, radical prostatectomy

Prostate cancer is the most commonly diagnosed solid organ malignancy among males and is the second leading cause of cancer-related deaths, accounting for approximately 30,000 deaths per year in the United States [1]. Preoperative risk stratification is crucial to guide the therapeutic decisionmaking process in prostate cancer. Clinical and pathological characteristics such as prostate specific antigen (PSA) level, clinical tumor stage, and Gleason score are currently used for prognosis and risk assessment. However, prostate cancer is a heterogeneous disease and the biological behavior can be evaluated only to a limited extent using these parameters. Some prostate cancer cases classified as low- and intermediate-risk

\footnotetext{
Abbreviations: BCR, biochemical recurrence; CI, confidence interval; IHC, immunohistochemistry; OR, odds ratio; PSA, prostate-specific antigen; Sema3s, class-3 semaphorins.
}

diseases show more aggressive behavior and more chance of recurrence after radical treatment $[2,3]$. Thus, identification of reliable biomarkers in prostate biopsy samples in parallel with other existing parameters for prediction of postoperative recurrence, especially for patients with low- or intermediaterisk disease, is urgently needed.

The class-3 semaphorin family members (Sema3A-F, Sema3s) is the only protein family comprised of secreted vertebrate semaphorins, which were initially identified to play an important role in axonal guidance and cell migration [4]. Interestingly, our previous studies show that Sema3s are also involved in the lymph node metastasis of prostate cancer, and are likely to modulate the behavior of cancers with a pro-tumoral or an anti-tumoral effect, depending on their subtypes [5-8]. Sema3A, 3B, 3D, and 3F act as anti-tumorigenic agents, since they increase apoptosis and inhibit cell migration 
by activating their specific receptors in multiple tumor cells, including prostate cancer [9-11], whereas Sema3C promotes angiogenesis and enhances invasive and metastatic characteristics [12]. In addition, Sema3E was shown to act both as a pro-angiogenic and an anti-angiogenic factor in vitro, either attracting or repelling endothelial cells expressing its receptor, PlexinD1 [5]. However, to our knowledge, no prior study has critically investigated the value of Sema3s expression in preoperative biopsy samples for the prediction of biochemical recurrence $(\mathrm{BCR})$ after radical prostatectomy. In this study, we evaluated Sema3s expression by immunohistochemistry (IHC) on 198 prostate biopsies with low- and intermediate-risk localized prostate cancer to specifically address two questions: (1) the frequency of positive Sema3s staining in prostate biopsies; (2) whether positive or negative Sema3s IHC is associated with the BCR rate after radical prostatectomy.

\section{Patients and methods}

Patients. This study was approved by the Institutional Review Board of the Third Affiliated Hospital of Sun Yat-sen University. Written informed consent was obtained from all patients. Between January 2007 and December 2010, a total of 235 cases were defined as low- and intermediate-risk prostate cancer according to the D'Amico risk group, i.e. clinical stage T1c or T2a or T2b, PSA $\leq 20 \mathrm{ng} / \mathrm{mL}$, Gleason score on biopsy $\leq 7$. [13]. All these 235 patients underwent laparoscopic radical prostatectomy as previously reported by one single surgeon (X.G.) [14]. Lymphadenectomy was usually performed in patients with intermediate risk. Those with positive surgical margin or positive lymph node were excluded. In total, 198 patients remained for the present analysis. Table 1 summarizes the clinical and pathological characteristics of patients included in this study.

For all patients enrolled, postoperative follow-up was performed quarterly in the first year, semiannually for the second year, and annually thereafter by clinical evaluation, measurement of total serum PSA, and by other measures as indicated. BCR was defined as two consecutive PSA levels of $>0.2 \mathrm{ng} / \mathrm{ml}$ after surgery [15]. Postoperative endocrine therapy was not administrated until BCR. The median follow-up was 42 months (range, 6-60) for all patients.

Hisopathologic analysis. All patients underwent at least 10-core transrectal ultrasound-guided biopsies, and at least 10 paraffin blocks per patient were prepared. The morphologic diagnosis was confirmed on H\&E slides by 2 independent urological pathologists (D.He and C.-K. Shao) who were blinded to the results of Sema3s staining IHC. Gleason score and morphologic feature of each case was assessed independent of Sema3s staining evaluation. If necessary, immunostaining for P504S, 34ßE12 and p63, was carried out using an avidin-biotin complex staining procedure as previously reported [16].

Evaluation of Sema3s protein expression via IHC. The biopsy paraffin blocks were available for analysis and all corresponding H\&E-stained and immunostaining slides were reviewed. A representative slide from each patient with carcinoma was selected for evaluation of Sema3s protein expression status by IHC. The selection of the core for IHC analysis was made by the pathologist conducting the diagnosis and the core with the highest proportion of cancer cells was elected. In brief, following deparaffinization, $5-\mu \mathrm{m}$ sections were dehydrated and blocked in $1 \%$ hydrogen peroxide in methanol for 20 minutes. Sections were pretreated using the microwave antigen retrieval procedure in $10 \mathrm{mmol} / \mathrm{L}$ of boiling EDTA ( $\mathrm{pH} \mathrm{8.0).} \mathrm{The} \mathrm{tissue} \mathrm{sections} \mathrm{were} \mathrm{then} \mathrm{incubated} \mathrm{with}$ primary antibodies against Sema3A (sc-28867, Santa Cruz Biotechnology Inc., CA, USA), Sema3B (sc-10720, Santa Cruz), Sema3C (sc-74555, Santa Cruz), Sema3D (sc-68043, Santa Cruz), Sema3E (sc-49733, Santa Cruz) and Sema3F (sc-68796, Santa Cruz) diluted to 1:100 in phosphate-buffered saline. The biotin-streptavidin detection system (Abcam Inc., MA, USA) was used for detecting the signals from the stained tissue sections. Samples were developed with aliquid diaminobenzidineandsubstrate-chromogen system (Abcam Inc.) and counterstained with hematoxylin to visualize the staining of the Sema3s protein. Following a hematoxylin counterstaining, positive immunostaining was scored by two independent experienced observers (L.-Y. Li and J. Pang) in a blinded fashion. Tumor specimens were scored in a semiquantitative manner with the following scale: $(0)$ less than $10 \%$ positive staining cells; (1+) 10-25\%; (2+) 25-50\%; (3+) more than 50\%. Cases with scores of $2+$ or $3+$ were designated as "positive," whereas cases with scores of 0 or $1+$ were designated as "negative" [17, 18].In case of any discrepant scores, the samples were further reviewed by another urological pathologist (Z.-L. Su), who acted as an arbiter. All 3 reviewers were unaware of patients' clinicopathological parameters.

Statistical analysis. Continuous parametric variables were reported as the median values and range. Mann-Whitney $U$-test and chi-square tests were used to compare continuous and categorical variables as appropriate. A logistic regression model, including age, PSA, clinical T stage, biopsy Gleason score, and Sema3s immunostaining, was used to perform univariable and multivariable analyses in a stepwise manner. The predictive values of Sema3s expression status were tested using Kaplan-Meier analysis with the Log-rank test for assessing differences. SPSS 16.0 software package (SPSS Inc., Chicago, IL,USA) was used for all the analyses and a two-tailed test with $P<0.05$ was considered statistically significant.

\section{Results}

Sema3s were diffusely expressed on cancer cells with membranous and/or cytoplasmic immunoreactivity in biopsy samples (Fig.1). Among these 198 cases, 34 with BCR were observed during a median follow-up of 42 months (range, 660 ). The overall BCR-free survival rate was $82.8 \%$. The positive percentages of Sema3A-F expression in the BCR group and the BCR-free group were listed in Table 1. Semiquantitative analysis revealed that the BCR-free group had significantly 

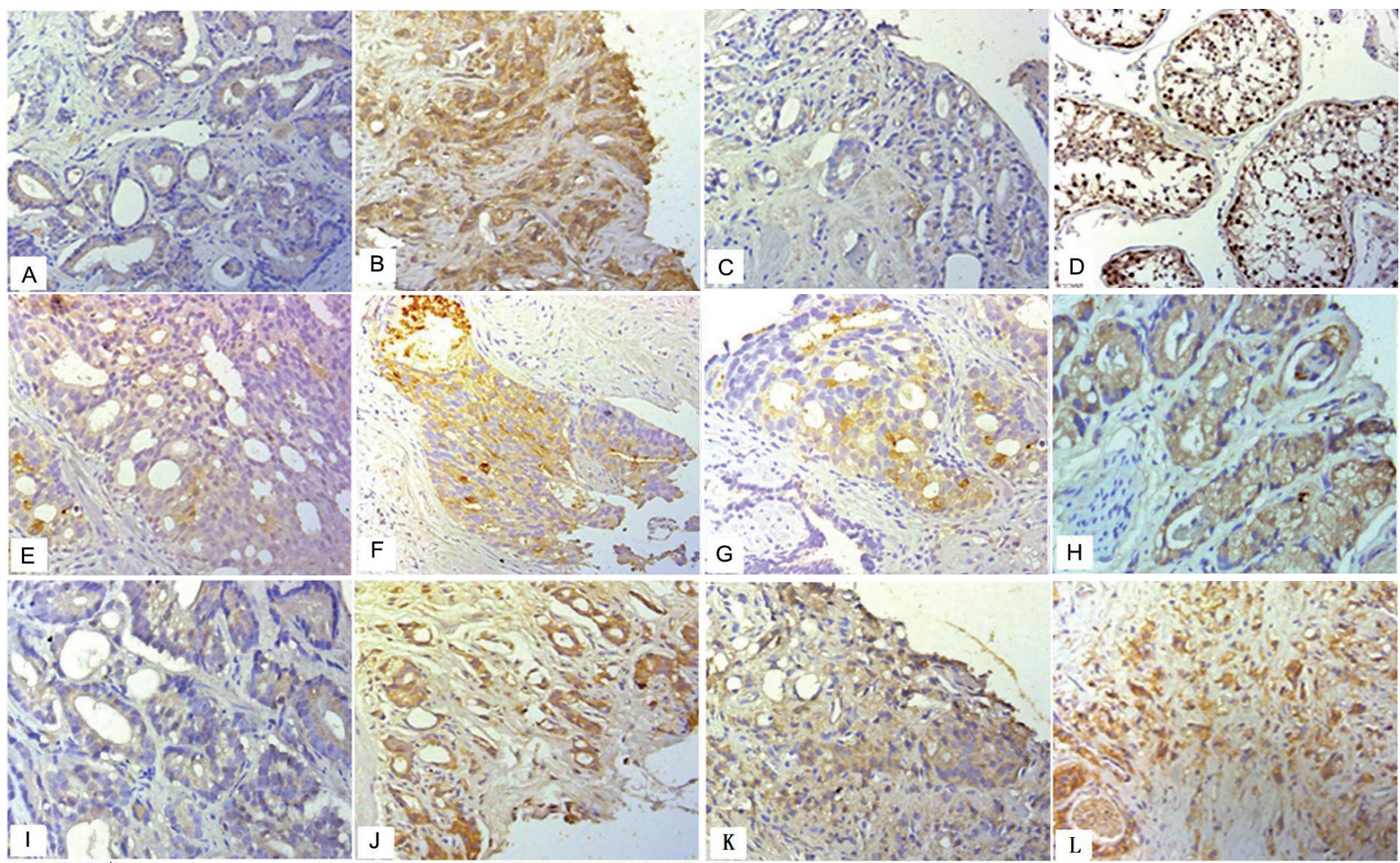

Figure 1. Representative images of the immunohistochemical staining of Sema3s proteins in biopsy samples: Sema3s are diffusely expressed on cancer cells with membranous and/or cytoplasmic immunoreactivity. A/B, negative/positive Sema3A expression; C/D, negative/positive Sema3B expression; E/F, negative/positive Sema3C expression; G/H, negative/positive Sema3D expression; I/J, negative/positive Sema3E expression; K/L, negative/ positive Sema3F expression. Original magnification of $\mathrm{H} \& \mathrm{E}$ images, $\times 200$.

higher positive percentages of Sema3A, Sema3B, and Sema3E, but had significantly lower expression of Sema3C, relative to the BCR group (Table $1 \&$ Fig. 1). The median relapse time was 17 months (range, 9-32). 11 cases with BCR occurred in 12 months, 14 cases had the relapse between 12 to 24 months, and 9 patients recurred 24 months later.

Table 2 summarizes the univariable and multivariable analyses for BCR. Parameters, including patient age at surgery, preoperative PSA levels, biopsy Gleason score, clinical T stage, and the expression status of Sema3s were included in these analyses using a logistic regression model. On univariable regression analysis, biopsy Gleason score (odds ratio [OR]: 2.49, $P=0.016$ ), clinical T stage (OR:2.31, $P=0.007$ ), Sema3A(OR:0.20, $P<0.001$ ), Sema3B(OR:0.48, $P=0.023$ ), Sema3E(OR:0.34, $P=0.02$ ), and Sema3C(OR:2.71, $P=0.003$ ) staining were significantly associated with $B C R$, while age and preoperative PSA level were not ( $P=0.39, P=0.21$, respectively). On multivariable analysis, biopsy Gleason score (OR:1.96, $P=0.033$ ), Sema3A(OR:0.19, $P<0.001)$, Sema3B(OR:0.38, $P=0.003)$, Sema3E(OR:0.39, $P=0.007)$, and Sema3C(OR:2.31, $P=0.014$ ) staining were independent predictors of $\mathrm{BCR}$ (Table 2).
Moreover, the actuarial probability of remaining free of BCR for this cohort of patients during the follow-up period after surgery was calculated using the Kaplan-Meier method. The BCR rates in Sema3A-negative, Sema3B-negative, and Sema3E-negative patients were significantly higher than those with Sema3A-positive, Sema3B-positive, and Sema3E-positive staining ( $P=0.001, P=0.003, P=0.037$, respectively, Log-rank test) (Fig. 2A, B and E). Patients with Sema3C-positive tumors were significantly more likely to experience BCR than those with Sema3C-negative tumors ( $P=0.029$, Log-rank test, Fig. 2C). However, Sem3D or Sema3F expression alone could not predict BCR with statistical significance $(P=0.71, P=0.36$, respectively, Log-rank test, Fig. 2D and F). In addition, we found that patients with concurrent Sema3C-positive and Sema3A-negative, Sema3B-negative, Sema3E-negative tumors had significantly more chance to experience BCR than those with other patterns of Sema3s staining $(P<0.001$, Log-rank test, Fig. $2 G)$.

\section{Discussion}

BCR is currently one of the major challenges in prostate cancer patients who undergo radical prostatectomy. Although 
Table 1. Characteristics of the 198 patients with low- and intermediate risk prostate cancer

\begin{tabular}{lccc}
\hline Characteristic & BCR & BCR-free & $P$ value \\
\hline Number of cases & 34 & 164 & \\
Median age (yr, range) & $64(47-73)$ & $63(50-71)$ & 0.131 \\
Preoperative PSA (ng/ml, range) & $9.8(2.2-16.5)$ & $10.2(5.2-19.6)$ & 0.215 \\
Clinical T stage, n (\%) & & & 0.053 \\
$\quad$ T1c & $10(29.4)$ & $67(40.9)$ & \\
T2a & $7(20.6)$ & $50(30.5)$ & \\
T2b & $17(50.0)$ & $47(28.6)$ & \\
Biopsy Gleason score, n (\%) & & & 0.028 \\
$\quad$ 6 & $14(41.2)$ & $101(61.6)$ & \\
7 & $20(58.8)$ & $63(38.4)$ & \\
Sema 3A expression, n (\%) & & & $<0.001$ \\
$\quad$ Negative & $29(85.3)$ & $75(45.7)$ & \\
$\quad$ Positive & $5(14.7)$ & $89(54.3)$ & \\
Sema 3B expression, n (\%) & & & 0.020 \\
$\quad$ Negative & $25(73.5)$ & $73(44.5)$ & \\
$\quad$ Positive & $9(26.5)$ & $91(54.5)$ & \\
Sema 3C expression, n (\%) & & & 0.045 \\
$\quad$ Negative & $11(32.4)$ & $84(51.2)$ & \\
Positive & $23(67.6)$ & $80(48.8)$ & \\
Sema 3D expression, n (\%) & & & 0.871 \\
$\quad$ Negative & $20(58.8)$ & $94(57.3)$ & \\
Positive & $14(41.2)$ & $70(42.7)$ & \\
Sema 3E expression, n (\%) & & & 0.007 \\
Negative & $24(70.6)$ & $74(45.1)$ & \\
$\quad$ Positive & $10(29.4)$ & $90(54.9)$ & \\
Sema 3F expression, n (\%) & & & \\
$\quad$ Negative & $13(38.2)$ & $75(45.7)$ & \\
Positive & $21(61.8)$ & $89(54.3)$ & \\
\hline
\end{tabular}

Abbreviations: BCR, biochemical recurrence; PSA, prostate-specific antigen.

histologic grading is one of the critical predictors of BCR, its use is limited because most patients have moderately-differentiated tumors (Gleason scores of 6 or 7) [19]. The use of appropriate tumor biomarkers, as supplements to clinical parameters, might improve the predictive value of biochemical and histological analyses [20,21]. However, there is still a lack of reliable biomarkers that can specifically and accurately reflect the likelihood of BCR after curative treatment. In the present study, we evaluated the value of Sema3s expression by IHC on preoperative biopsy samples for the prediction of BCR after radical prostatectomy. Our results suggest that patients with Sema3C-positive and Sema3A-negative, 3Bnegative, $3 \mathrm{E}$-negative tumors had significantly more chance to experience BCR.

Prior studies demonstrated that Sema3s not only played a critical role in axon guidance, but also could regulate the response of cancer cells and endothelial cells to either promote or inhibit tumor angiogenesis and progression through various mechanisms [5, 6]. Yacoub et al. [22] evaluated 120 patients with localized prostate cancer who were treated with radical prostatectomy and found that expression of Sema3A was associated with lower preoperative PSA and pathological stage. Moreover, their analysis revealed that dysregulation of Sema3A plays a key role in the progression of prostate cancer and suggested a loss of the inhibitory Sema3A autocrine loop in hormone-refractory prostate cancer [22]. Similar results were described by Herman et al. [23], who suggested that overexpression of Sema3A decreased prostate cancer invasion and adhesion in vitro. In contrast, tumors in prostate cancer patients with high Sema3C expression possessed higher invasive and metastatic characteristics [23]. Consistent with these studies, we found that Sema3C-positive and Sema3A-negative expressions were associated with a higher probability of BCR. Furthermore, the multivariate analysis showed that Sema3Anegative and Sema3C-positive expressions were independent risk factors for predicting relapse.

Sema3B and 3F were initially characterized as tumor suppressors whose functions are lost in carcinoma cells by allelic loss or tumor-acquired promoter methylation. Sema3B can induce apoptosis and its pro-apoptotic effect is inhibited by VEGF [24]. In addition, Sema3F can affect tumor cell behavior

Table 2. Univariable and multivariable analyses for biochemical recurrence

\begin{tabular}{|c|c|c|c|c|}
\hline \multirow{2}{*}{ Variable } & \multicolumn{2}{|c|}{ Univariable analysis } & \multicolumn{2}{|c|}{ Multivariable analysis } \\
\hline & OR $(95 \% \mathrm{CI})$ & $P$ & OR $(95 \% \mathrm{CI})$ & $P$ \\
\hline Age: $<65 \mathrm{yr}, \geq 65 \mathrm{yr}$ & $0.98(0.96-1.02)$ & 0.39 & - & - \\
\hline Preoperative PSA (ng/ml): $<10,10-20$ & $1.47(0.80-2.73)$ & 0.21 & - & - \\
\hline Biopsy Gleason score: 6, 7 & $2.49(1.15-3.93)$ & 0.016 & $1.96(1.06-3.62)$ & 0.033 \\
\hline Clincal $\mathrm{T}$ stage: $\mathrm{T} 1 \mathrm{c}-\mathrm{T} 2 \mathrm{a}, \mathrm{T} 2 \mathrm{~b}$ & $2.31(1.26-4.21)$ & 0.007 & $1.24(1.02-2.93)$ & 0.091 \\
\hline Sema 3A: positive, negative & $0.20(0.09-0.44)$ & $<0.001$ & $0.19(0.089-0.42)$ & $<0.001$ \\
\hline Sema 3B: positive, negative & $0.48(0.26-0.91)$ & 0.023 & $0.38(0.19-0.72)$ & 0.003 \\
\hline Sema 3C: positive, negative & $2.71(1.41-5.21)$ & 0.003 & $2.31(1.19-4.49)$ & 0.014 \\
\hline Sema 3D: positive, negative & $0.91(0.49-1.68)$ & 0.77 & - & - \\
\hline Sema 3E: positive, negative & $0.34(0.18-0.66)$ & 0.02 & $0.39(0.20-0.78)$ & 0.007 \\
\hline Sema 3F: positive, negative & $0.93(0.51-1.69)$ & 0.81 & - & - \\
\hline
\end{tabular}

Abbreviations: OR, odds ratio; CI, confidence interval; PSA, prostate-specific antigen. 

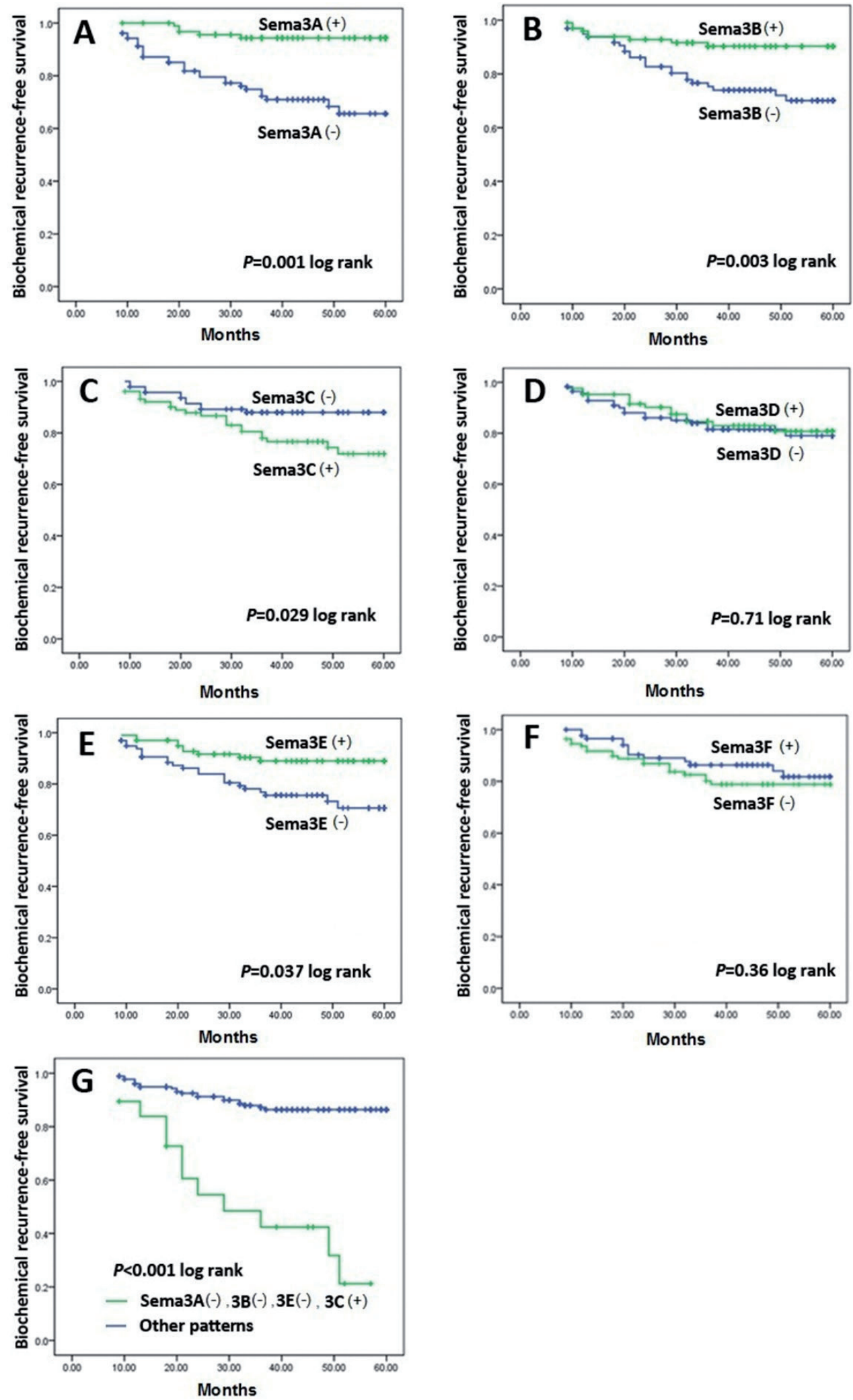

Figure 2. Association between the Sema3s protein expression and biochemical recurrence: Kaplan-Meier analysis showed that patients with Sema3A-negative, Sema3B-negative, Sema3E-negative and Sema3C-positive tumors (each alone or four markers combined) were significantly more likely to experience biochemical recurrence $(\mathrm{A}, \mathrm{B}, \mathrm{C}, \mathrm{E}, \mathrm{G})$, while Sem3D or Sema3F expression alone could not predict biochemical recurrence with statistical significance(D,F). 
directly and has ability to suppress the metastatic spread of cells from tumors [25]. Overexpression of both Sema3B and $3 \mathrm{~F}$ is negatively associated with tumor progression. Beuten et al. [26] performed a case-controlled study of 789 prostate cancer cases and found that polymorphisms in Sema3B and 3F were associated with a high Gleason score (OR 1.44, $P=0.021)$ and correlated with increased prostate cancer risk and poor prognosis (OR 2.72, $P=0.016$ and OR 3.32, $P=0.02$, respectively). However, our present findings showed only Sema3B was independently predictive of recurrence-free survival in patients with low- and intermediate-risk prostate cancer. The role of Sema3F in predicting BCR need to be evaluated in further prospective studies.

Sema3E is considered unique among Sema3s in that it binds directly to the plexin-D1 receptor, but not to neuropilins [27]. Plexin-D1 can transduce anti-angiogenic signals and is up-regulated in tumor-associated blood vessels [28]. It also forms complexes with neuropilins. Whether the Sema3E secreted by tumor cells promotes or inhibits cancer growth may depend on the constitution of the receptor complex to which it binds on the tumor cells. Prostate tumors express high levels of neuropilin-1, and this overexpression correlates with advanced disease and a high Gleason score [29]. Blanc et al. [30] showed that strong staining for Sema3E was observed in $52 \%$ of prostate cancer tumor tissue samples, compared with $11 \%$ in non-malignant tissue samples $(P=0.0001)$. The fact that $43 \%$ of tumor samples had a greater intensity of Sema3E staining, compared to non-malignant tissues from the same patient, suggests that Sema3E is overexpressed in prostate cancer. Our present data corroborated this association and demonstrated that Sema3E was negatively correlated with the risk of BCR after radical prostatectomy. Meanwhile, Kigel et al. [11] showed that Sema3D can function as a potent antitumorigenic agent, and could inhibit tumor development and attenuate angiogenesis. Interestingly, although $58.8 \%$ of cases with BCR in our study had Sema3D-negative staining, Sema3D expression alone was not an independent predictive factor for BCR.

In this study, recurrent-free survival plot analysis confirmed that positive staining of Sema3A, 3B, and 3E and negative Sema3C staining were associated with a favorable prognosis for patients with low- and intermediate-risk prostate cancer who underwent radical prostatectomy. In multivariate analysis, Sema3A, 3B, 3C, and 3E staining were shown to be independent risk factors for predicting $\mathrm{BCR}$. We propose that the expression status of Sema3A, 3B, 3C, and 3E in biopsy samples may supplement the current prognostic factors, i.e. clinical or pathological stage, Gleason score and PSA, as predictors of $B C R$. We note that even with relatively short follow up in our study, the BCR rate (17.2\%) is higher than those reported by some authors [15]. In our opinion, this may be due to the high proportion of patients with intermediate-risk prostate cancer (about 60\%) included in our cohort.

Some limitations of this study should be noted. Firstly, our study was in a retrospective fashion; and secondly, the number of evaluated patients was not particularly large, which might limit the statistical power of the predictive value of some markers. Consequently, further prospective studies in larger patient populations are needed to validate the current observations.

In summary, we showed that Sema3A, 3B, 3C, and 3E immunostaining in prostate biopsies, as supplements to clinicopathological parameters, could be used for predicting BCR in low- and intermediate-risk prostate cancer patients after radical prostatectomy. Specially, concurrent Sema3C-positive and Sema3A-negative, 3B-negative, 3E-negative staining is associated with an adverse prognosis.

Acknowledgements: This study was supported by Program of 5010 of Sun-Yat Sen University (2007028 to X.G.), Key Project of Chinese Ministry of Health (to X.G.), National Natural Science Foundation of China (30772178 to X.G. and 81001139 to L.Y.L), the Science and Technology Foundation of the Guangdong Province (2012A030400009 to X.G.), and the Fundamental Research Funds for the Central Universities (11ykpy39 to L.Y.L and to X.G.).

\section{References}

[1] SIEGEL R, WARD E, BRAWLEY O, JEMAL A. Cancer statistics, 2011: the impact of eliminating socioeconomic and racial disparities on premature cancer deaths. CA Cancer J Clin 2011; 61: 212-36. http: //dx.doi.org/10.3322/caac.20121

[2] NOGUCHI M, STAMEY TA, MCNEAL JE, YEMOTO CM. Relationship between systematic biopsies and histological features of 222 radical prostatectomy specimens: lack of prediction of tumor significance for men with nonpalpable prostate cancer. J Urol 2001; 166: 104-9; discussion 9-10. http: //dx.doi.org/10.1016/S0022-5347(05)66086-7

[3] GUMULEC J, MASARIK M, KRIZKOVA S, HLAVNA M, BABULA P, et al. Evaluation of alpha-methylacyl-CoA racemase, metallothionein and prostate specific antigen as prostate cancer prognostic markers. Neoplasma 2012; 59: 191-201. http: //dx.doi.org/10.4149/neo 2012 025

[4] BIELENBERG DR, SHimizU A, KLAGSBRUN M. Semaphorin-induced cytoskeletal collapse and repulsion of endothelial cells. Methods Enzymol 2008; 443: 299-314. http: //dx.doi.org/10.1016/S0076-6879(08)02015-6

[5] NEUFELD G, KESSLER O. The semaphorins: versatile regulators of tumour progression and tumour angiogenesis. Nat Rev Cancer 2008; 8: 632-45. http: //dx.doi.org/10.1038/nrc2404

[6] STATON CA. Class 3 semaphorins and their receptors in physiological and pathological angiogenesis. Biochem Soc Trans 2011; 39: 1565-70. http: //dx.doi.org/10.1042/ BST20110654

[7] PANG J, LIU W-P, LIU X-P, LI L-Y, FANG Y-Q, et al. Profiling Protein Markers Associated with Lymph Node Metastasis in Prostate Cancer by DIGE-based Proteomics Analysis. Journal of Proteome Research 2010; 9: 216-26. http: //dx.doi. org/10.1021/pr900953s

[8] GAO X, PANG J, LI LY, LIU WP, DI JM, et al. Expression profiling identifies new function of collapsin response mediator protein 4 as a metastasis-suppressor in prostate cancer. 
Oncogene 2010; 29: 4555-66. http: //dx.doi.org/10.1038/ onc. 2010.213

[9] TOMIZAWA Y, SEKIDO Y, KONDO M, GAO B, YOKOTA $\mathrm{J}$, et al. Inhibition of lung cancer cell growth and induction of apoptosis after reexpression of 3p21.3 candidate tumor suppressor gene SEMA3B. Proc Natl Acad Sci U S A 2001; 98: 13954-9. http: //dx.doi.org/10.1073/pnas.231490898

[10] ROCHE J, BOLDOG F, ROBINSON M, ROBINSON L, VARELLA-GARCIA M, et al. Distinct 3p21.3 deletions in lung cancer and identification of a new human semaphorin. Oncogene 1996; 12: 1289-97.

[11] KIGEL B, VARSHAVSKY A, KESSLER O, NEUFELD G. Successful inhibition of tumor development by specific class-3 semaphorins is associated with expression of appropriate semaphorin receptors by tumor cells. PLoS One 2008; 3: e3287. http: //dx.doi.org/10.1371/journal.pone.0003287

[12] NEUFELD G, SHRAGA-HELED N, LANGE T, GUTTMANNRAVIV N, HERZOG Y, et al. Semaphorins in cancer. Front Biosci 2005; 10: 751-60. http: //dx.doi.org/10.2741/1569

[13] D‘AMiCO AV, WHITTINGTON R, MALKOWICZ SB, SCHULTZ D, BLANK K, et al. Biochemical outcome after radical prostatectomy, external beam radiation therapy, or interstitial radiation therapy for clinically localized prostate cancer. JAMA 1998; 280: 969-74. http://dx.doi.org/10.1001/ jama.280.11.969

[14] GAO X, JIAN-HUA Z, LI L-Y, QIU J-G, PU X-Y. Laparoscopic radical prostatectomy: oncological and functional results of 126 patients with a minimum 3 -year follow-up at a single Chinese institute. Asian Journal of Andrology 2009; 11: 548-56. http: //dx.doi.org/10.1038/aja.2009.42

[15] MOTTET N, BELLMUNT J, BOLLA M, JONIAU S, MASON M, et al. EAU guidelines on prostate cancer. Part II: Treatment of advanced, relapsing, and castration-resistant prostate cancer. Eur Urol 2011; 59: 572-83. http: //dx.doi. org/10.1016/j.eururo.2011.01.025

[16] GAO X, LI L-Y, ZHOU F-J, XIE K-J, SHAO C-K, et al. ERG Rearrangement for Predicting Subsequent Cancer Diagnosis in High-Grade Prostatic Intraepithelial Neoplasia and Lymph Node Metastasis. Clinical Cancer Research 2012; 18: 4163-72. http: //dx.doi.org/10.1158/1078-0432.CCR-11-2449

[17] PEI H, ZHU H, ZENG S, LI Y, YANG H, et al. Proteome analysis and tissue microarray for profiling protein markers associated with lymph node metastasis in colorectal cancer. J Proteome Res 2007; 6: 2495-501. http://dx.doi.org/10.1021/ pr060644r

[18] CUI Y, WU J, ZONG M, SONG G, JIA Q, et al. Proteomic profiling in pancreatic cancer with and without lymph node metastasis. Int J Cancer 2009; 124: 1614-21. http: //dx.doi. org/10.1002/ijc. 24163
[19] DVORACKOVA J, UVIROVA M. A molecularly genetic determination of prognostic factors of the prostate cancer and their relationships to expression of protein p27kip1. Neoplasma 2007; 54: 149-54.

[20] SUN YF, YANG XR, ZHOU J, QIU SJ, FAN J, et al. Circulating tumor cells: advances in detection methods, biological issues, and clinical relevance. J Cancer Res Clin Oncol 2011; 137: 1151-73. http: //dx.doi.org/10.1007/s00432-011-0988-y

[21] NOGUEIRA L, CORRADI R, EASTHAM JA. Other biomarkers for detecting prostate cancer. BJU Int 2010; 105: 166-9. http: //dx.doi.org/10.1111/j.1464-410X.2009.09088.x

[22] YACOUB M, COULON A, CELHAY O, IRANI J, CUSSENOT $\mathrm{O}$, et al. Differential expression of the semaphorin 3A pathway in prostatic cancer. Histopathology 2009; 55: 392-8. http: //dx.doi.org/10.1111/j.1365-2559.2009.03406.x

[23] HERMAN JG, MEADOWS GG. Increased class 3 semaphorin expression modulates the invasive and adhesive properties of prostate cancer cells. Int J Oncol 2007; 30: 1231-8.

[24] CASTRO-RIVERA E, RAN S, THORPE P, MINNA JD. Semaphorin 3B (SEMA3B) induces apoptosis in lung and breast cancer, whereas VEGF165 antagonizes this effect. Proc Natl Acad Sci U S A 2004; 101: 11432-7. http: //dx.doi. org/10.1073/pnas.0403969101

[25] NASARRE P, CONSTANTIN B, ROUHAUD L, HARNOIS T, RAYMOND G, et al. Semaphorin SEMA3F and VEGF have opposing effects on cell attachment and spreading. Neoplasia 2003; 5: 83-92.

[26] BEUTEN J, GARCIA D, BRAND TC, HE X, BALIC I, et al. Semaphorin $3 \mathrm{~B}$ and $3 \mathrm{~F}$ single nucleotide polymorphisms are associated with prostate cancer risk and poor prognosis. J Urol 2009; 182: 1614-20. http: //dx.doi.org/ 10.1016/j.juro.2009.06.016

[27] GU C, YOSHIDA Y, LIVET J, REIMERT DV, MANN F, et al. Semaphorin 3E and plexin-D1 control vascular pattern independently of neuropilins. Science 2005; 307: 265-8. http: //dx.doi.org/10.1126/science.1105416

[28] GITLER AD, LU MM, EPSTEIN JA. PlexinD1 and semaphorin signaling are required in endothelial cells for cardiovascular development. Dev Cell 2004; 7: 107-16. http: //dx.doi. org/10.1016/j.devcel.2004.06.002

[29] LATIL A, BIECHE I, PESCHE S, VALERI A, FOURNIER G, et al. VEGF overexpression in clinically localized prostate tumors and neuropilin-1 overexpression in metastatic forms. Int J Cancer 2000; 89: 167-71. http://dx.doi.org/10.1002/(SICI)10970215(20000320)89: 2<167: : AID-IJC11>3.0.CO; 2-9

[30] BLANC V, NARICULAM J, MUNSON P, FREEMAN A, KLOCKER $\mathrm{H}$, et al. A role for class 3 semaphorins in prostate cancer. Prostate 2011; 71: 649-58. http: //dx.doi.org/10.1002/ pros.21281 